Transport and Communications Science Journal, Vol 71, Issue 2 (02/2020), 154-166

Transport and Communications Science Journal
ARTIFICIAL NEURAL NETWORK BASED MODELING OF THE
AXIAL CAPACITY OF RECTANGULAR CONCRETE FILLED
STEEL TUBES

\author{
Hai-Bang Ly*, Thuy-Anh Nguyen
}

University of Transport Technology, 54 Trieu Khuc, Thanh Xuan, Hanoi, Vietnam

ARTICLE INFO

TYPE: Research Article

Received: $13 / 02 / 2020$

Revised: 28/02/2020

Accepted: 28/02/2020

Published online: 29/02/2020

https://doi.org/10.25073/tcsj.71.2.10

* Corresponding author

Email: banglh@utt.edu.vn; Tel: 0966661185

Abstract. Concrete filled steel tubes (CFST) with many advantages compared with conventional structural members made by steel or reinforced concrete has been widely applied in the field of civil engineering. The axial capacity of CFST, which is considered as the most important mechanical property, depends on many constituting factors such as the mechanical properties of materials or the cross-section of the tubes. In this study, the Artificial Neural Network (ANN) with the Levenberg-Marquardt algorithm is used to predict the axial capacity of CFST with rectangular cross-section. A number of 99 samples, collected from published international studies, is divided into two parts: the training part (69 samples), used to construct the BPNN black-box, and 30 samples in the testing part to evaluate the performance of the BPNN model. The main input parameters used in this study are the height of steel tube, width of steel tube, the thickness of steel tube, length of the column, the yield stress of steel and the compressive strength of concrete. The results show that the ANN algorithm is a good predictor for the problem with low error and high correlation $(\mathrm{R}=0.99)$ between the experimental and predicted results derived from the ANN algorithm.

Keywords: concrete filled steel tube, artificial neural network, axial compression, axial capacity.

(C) 2020 University of Transport and Communications 


\section{NGHIÊN CÚU DỬ BÁO SỨC CHỊU TẢI TỚI HẠN CỦA CẤU KIỆN CộT ỐNG THÉP NHỒI BÊ TÔNG CÓ TIẾT DIỆN HİNH CHŨ NHẬT BẦNG MẠNG NƠ RON NHÂN TẠO}

\section{Lý Hải Bằng*, Nguyễn Thùy Anh}

Trường Đại học Công nghệ Giao thông Vận tải, Số 54 Triều Khúc, Thanh Xuân, Hà Nội, Việt Nam

THÔNG TIN BÀI BÁO

CHUYÊN MỤC: Công trình khoa học

Ngày nhận bài: 13/02/2020

Ngày nhận bài sửa: 28/02/2020

Ngày chấp nhận đăng: 28/02/2020

Ngày xuất bản Online: 29/02/2020

https://doi.org/10.25073/tcsj.71.2.10

* Tác giả liên hệ

Email: banglh@utt.edu.vn; Tel: 0966661185

Tóm tắt. Kết cấu ống thép nhồi bê tông (CFST) có nhiều lợi thế so với kết cấu thông thường làm bằng thép hoặc bê tông cốt thép nên hiện nay đang được sử dụng rộng rãi trong lĩnh vực xây dựng công trình. Khả năng chịu lực dọc trục $(\mathrm{Pu})$ của CFST là một trong những tính chất cơ học quan trọng nhất và phụ thuộc vào nhiều yếu tố như thuộc tính của vật liệu, kích thước hình học mặt cắt. Trong nghiên cứu này, mô hình mạng Nơ-Ron lan truyền ngược (BPNN) với việc sử dụng thuật toán Levenberg - Marquardt được sử dụng để dự đoán sức chịu tải tới hạn $(\mathrm{Pu})$ của cột CFST hình chữ nhật. Với 99 dữ liệu thử nghiệm từ các công trình đã công bố, 69 số liệu đã được chọn để huấn luyện và 30 số liệu được sử dụng để kiểm chứng mô hình BPNN. Các thông số chính được nghiên cứu trong bài viết gồm chiều cao, chiều rộng mặt cắt, độ dày của cột thép, chiều dài cột, cường độ của thép, cường độ bê tông đã được đề cập để dự đoán khả năng chịu lực tới hạn của cột CFST. Kết quả cho thấy mô hình ANN dự báo rất tốt với độ chính xác cao và sai số thấp (hệ số tương quan $\mathrm{R}=0.99$ ).

Từ khóa: Cột CFST mặt cắt chữ nhật, mạng nơ ron nhân tạo (ANN), tải trọng dọc trục, sức chịu tải tới hạn.

(C) 2020 Truờng Đại học Giao thông vận tải

\section{1. ĐẶT VẤN ĐỀ}

Ngày nay, thép kết cấu đã và đang được ứng dụng rộng rãi trong lĩnh vực công nghiệp, xây dựng bởi các những đặc tính ưu việt của nó như khả năng chịu lực lớn, độ tin cậy cao, cho 
phép biến dạng dẻo tốt. Tuy nhiên, thép kết cấu có nhược điểm là dễ bị gỉ và ăn mòn, giá thành cao. Hơn nữa, kết cấu ống thép cũng dễ bị biến dạng khi bị va đập. Vì vậy, việc nhồi bê tông vào ống thép là một giải pháp tăng cường khả năng chống ăn mòn của bề mặt bên trong của ống thép, tăng độ ổn định cục bộ của thành ống và tăng khả năng chống biến dạng. Loại kết cấu này được gọi là cột ống thép nhồi bê tông (Concrete Filled Steel Tube - CFST).

Cho đến nay, cột ống thép nhồi bê tông đã được sử dụng rộng rãi trong việc xây dựng các tòa nhà nói chung và trong ngành giao thông là các cầu hiện đại, ngay cả trong các khu vực có địa chấn cao. Ở Việt Nam, kết cấu vòm ống thép nhồi bê tông đã được áp dụng tại nhiều công trình trong ngành giao thông vận tải như cầu Ông Lớn, cầu Xóm Củi, cầu Cần Giuộc hay cầu Đông Trù. Loại kêt cấu này đã thể hiện một số lợi thế so với cột thép rỗng và cột bê tông cốt thép, chẳng hạn như khả năng chịu lực dọc trục cao, độ dẻo và độ bền tốt, khả năng hấp thụ năng lượng lớn, xây dựng thuận tiện, tiết kiệm vật liệu và khả năng chống cháy cao. Bên cạnh đó, công trình xây dựng nhanh hơn do không cần ván khuôn, chi phí kết cấu thấp, bảo tồn môi trường do có thể tái sử dụng ống thép, sử dụng bê tông chất lượng cao với cốt liệu tái chế và độ tin cậy của việc sử dụng các cấu trúc CFST cho các tòa nhà chịu động đất cũng được tăng lên. Các nghiên cứu chỉ ra rằng kết cấu CFST tỏ ra hiệu quả cao khi chịu nén [1]. Vì vậy, mặt cắt ngang của cột $\mathrm{CFST}$ được chọn thường đối xứng như hình tròn, hình vuông hoặc hình chữ nhật. Trong số đó, các ống thép được đổ bê tông (CFST) với hình dạng vuông và hình chữ nhật mang lại lợi ích trong xây dựng, lý do là dễ dàng hơn trong việc kết nối giữa thiết kế mối nối dầm với cột và có độ cứng uốn cao hơn [2]. So với cột CFST vuông, cột hình chữ nhật có độ cứng uốn không đều dọc theo các trục khác nhau, vì vậy phù hợp với một số bộ phận của công trình như sườn vòm, mố cầu, trụ cầu, và một số loại kết cấu khác chịu lực không đều theo hai phương. Trong kỹ thuật xây dựng, khả năng chịu lực của cột CFST là một tham số thiết kế cơ bản và quan trọng, vì vậy cần phải có nghiên cứu về khả năng chịu lực của loại kết cấu này.

Hiện nay, các quy định về tính toán cột CFST đã được đề xuất trong các tiêu chuẩn thiết kế như AISC-LRFD, ACI 318-05, Viện Kiến trúc Nhật Bản, Tiêu chuẩn Châu Âu EC 4, Tiêu chuẩn Anh BS 5400 và Tiêu chuẩn Úc AS-5100.6. Ngoài ra, nhiều nghiên cứu thử nghiệm và mô phỏng số đã được thực hiện trên các tính chất cơ học của cột CFST hình chữ nhật dưới tác dụng của tải trọng dọc trục. Ví dụ, trong nghiên cứu của Lue và các cộng sự [3], 24 mẫu cột CFST hình chữ nhật có cường độ chịu nén của bê tông ( $\left.\mathrm{f}_{\mathrm{c}}{ }^{\prime}\right)$ thay đổi từ $29 \mathrm{MPa}$ đến $84 \mathrm{MPa}$ chịu tác dụng của tải trọng dọc trục đã được thử nghiệm. Kết quả thử nghiệm đã được so sánh với các mã thiết kế AISC-LRFD, EC 4, AS-5100 cho thấy mô hình có thể ước tính chặt chẽ khả năng tải dọc trục của các cột $\mathrm{CFST}$ hình chữ nhật cường độ cao và cường độ bình thường. Nghiên cứu của Han [4] sử dụng 24 mẫu cột hình chữ nhật với hai thông số chính là hệ số tỷ lệ giữa thép với bê tông và tỷ lệ giữa các cạnh của mặt cắt để xác định khả năng chịu lực của cột. Và còn nhiều nghiên cứu thử nghiệm khác liên quan đến các yếu tố ảnh hưởng đến khả năng chịu lực của các cột CFST hình chữ nhật như ảnh hưởng của đầm nén [5], điều kiện tải và điều kiện biên [6]. Bên cạnh đó, phân tích phần tử hữu hạn hiện cũng được sử dụng thường xuyên cho các vấn đề thiết kế và nghiên cứu [7], [8] nhờ sự tồn tại của nhiều phần mềm thương mại như ABAQUS và ANSYS. Tuy nhiên, cho đến nay, không có phương pháp được đề xuất nào ở trên được sử dụng rộng rãi do giới hạn phạm vi ứng dụng. Liu và cộng sự [911] đã tiến hành thử nghiệm các cột CFST có mặt cắt hình chữ nhật chịu tải trọng dọc trục và so sánh kết quả với các tiêu chuẩn như EC4 [12], AISC 360 [13] và ACI [14,15]. Tác giả rút ra kết luận rằng các tiêu chuẩn đã đánh giá thấp khả năng chịu lực của cột $\mathrm{CFST}$, ví dụ tiêu chuẩn EC4 chênh lệch $6 \%$ trong khi AISC và $\mathrm{ACI}$ đánh giá thấp sức chịu tải lần lượt là $16 \%$ và $14 \%$. Ngoài ra, Ma và Zhang [16] chỉ ra rằng các lý thuyết khác nhau tồn tại trong các tiêu 
chuẩn khác nhau mang lại kết quả khác nhau trong việc tính toán độ bền của các cột CFT hình chữ nhật. Nguyên nhân sai khác giữa các công thức tiêu chuẩn và thực nghiệm có thể kể đến như: khiếm khuyết hình học của ống thép trong quá trình sản xuất; ứng suất dư trong ống thép sau quá trình hàn hoặc lắp ráp; sự biến thiên của cường độ, độ cứng thực tế so với cường độ, độ cứng của vật liệu cấu thành cấu kiện; độ võng ban đầu tại vị trí giữa dọc trục các ống thép, chất lượng bê tông. Do đó, một phương pháp tổng quát và chính xác cần được phát triển để thiết kế các cột CFST hình chữ nhật.

Trong những năm gần đây, với sự phát triển nhanh chóng của các kỹ thuật trí tuệ nhân tạo, các thuật toán học máy (Machine learning algorithms - MLAs) đã được phổ biến trong mọi lĩnh vực của cuộc sống [17-19]. Mô hình mạng nơ-ron nhân tạo (Artificial Neural Network - ANN) được coi là công cụ mạnh để giải quyết các bài toán có tính phi tuyến, phức tạp và đặc biệt trong các trường hợp mà mối quan hệ giữa các yếu tố đầu vào và đầu ra không dễ thiết lập một cách tường minh. Một ưu điểm vượt trội của mô hình mạng nơron nhân tạo là khả năng tự học và điều chỉnh các trọng số để kết quả tính toán phù hợp với thực tế mà không phụ thuộc vào các phương trình cơ, lý hóa, hay ý kiến chủ quan.

Do đó, mục đích chính của bài viết này là nghiên cứu ứng dụng mô hình $\mathrm{ANN}$ trong việc dự đoán khả năng chịu lực dọc trục tới hạn của cột CFST hình chữ nhật.

\section{MÔ PHỎNG VÀ THỰC NGHIỆM}

\subsection{Công cụ dự báo dựa trên Mạng No-Ron nhân tạo (ANN)}

Mạng lưới thần kinh nhân tạo (ANN) là một sự trừu tượng hóa chức năng của các cấu trúc thần kinh sinh học của não người. Đây là một phương pháp tính toán mềm hiệu quả để giải quyết các vấn đề quá phức tạp được mô hình hóa và giải quyết bằng toán học cổ điển và các phương pháp truyền thống. Mạng nơ ron lan truyền ngược (BPNN), một phương pháp tìm nghiệm của ANN, thường được sử dụng để phân tích hồi quy và các ứng dụng thực tế. Cấu trúc mạng lan truyền ngược là sự kết hợp của các lớp khác nhau, trong đó lớp đầu tiên là lớp đầu vào và lớp cuối cùng là lớp đầu ra, các lớp ở giữa là các lớp ẩn được kết nối với cả hai lớp đầu vào và đầu ra, được minh họa trên hình 1 .

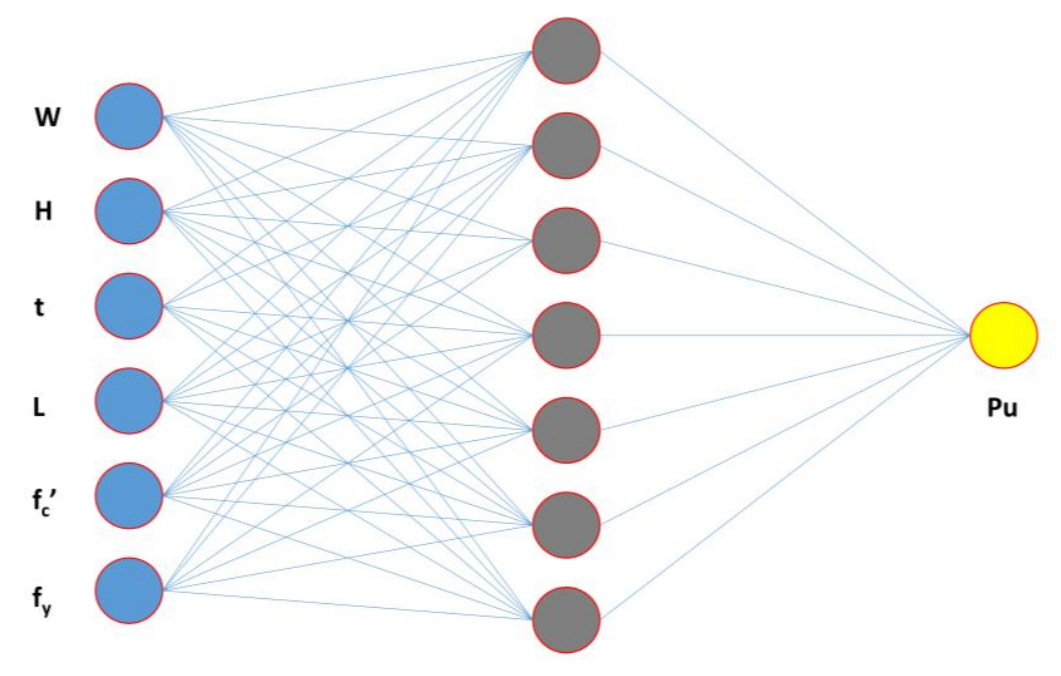

Lớp đầu vào

Lớp ẩn

Lớp đầu ra

Hình 1. Cấu trúc điển hình của mạng ANN được sủ dụng trong nghiên cứu này. 
Các lớp ẩn được tạo thành từ các đơn vị thần kinh hay còn gọi là các nơ ron. Các lớp này có trách nhiệm nhận đầu vào và xử lý chúng để tạo đầu ra. Số lượng các lớp ẩn trong mạng được xác định bởi mức độ phức tạp của vấn đề; mạng ban đầu được xây dựng với một số lượng nhỏ các lớp ẩn. Hiệu suất của mạng có thể được tối ưu hóa bằng cách tăng hoặc giảm số lượng tế bào thần kinh ẩn (số nơ ron) để có được số lượng tối ưu. Trong quá trình đào tạo, tín hiệu đầu vào truyền qua mạng theo hướng thuận, từ trái sang phải trên cơ sở từng lớp, tạo ra một tập hợp các giá trị trên các đơn vị đầu ra và sửa tất cả các trọng số của mạng. Sau đó, sự khác biệt giữa thực tế và các giá trị đầu ra mong muốn được đo và cường độ kết nối mô hình mạng được thay đổi để các đầu ra do mạng tạo ra trở nên gần hơn với các đầu ra mong muốn. Điều này đạt được bằng cách truyền ngược trong đó các thay đổi kết nối được truyền trở lại qua mạng bắt đầu bằng các kết nối đến lớp đầu ra và kết thúc với các kết nối đến lớp đầu vào. Tuy nhiên, trong các mạng BPNN truyền thống, có một số thiếu sót, chẳng hạn như tốc độ hội tụ chậm và dễ rơi vào mức tối thiểu cục bộ. Để khắc phục các nhược điểm đó, thuật toán Levenberg-Marquardt (LM) kết hợp giữa thuật toán giảm độ dốc và thuật toán Newton đã giảm đáng kể số lần lặp, tăng tốc độ hội tụ và có độ chính xác cao hơn. Đặc biệt, có tốc độ hội tụ nhanh nhất trong tất cả các BPNN truyền thống và cải tiến khác cho các mạng cỡ trung bình. Trong những năm gần đây, các BPNN cải tiến bằng thuật toán LM đã được sử dụng rộng rãi trong các lĩnh vực đánh giá và dự báo và cho thấy hiệu quả tốt. Để có được kết quả tốt hơn cho thử nghiệm, bài viết này đã chọn mô hình mạng thần kinh BP ba lớp được cải tiến dựa trên thuật toán LM để dự đoán sức chịu tải tới hạn của cột CFST.

Thuật toán Levenberg - Marquest được thiết kế đặc biệt để giảm thiểu các hàm lỗi tổng bình phương, có dạng:

$$
E=\frac{1}{2} \sum k\left(e_{k}\right)^{2}=\frac{1}{2}\|e\|^{2}
$$

Trong đó $\mathrm{e}_{\mathrm{k}}$ là lỗi trong mẫu thứ $\mathrm{k}$ và e là một vectơ có phần tử là $\mathrm{e}_{\mathrm{k}}$. Nếu sự khác biệt giữa vectơ trọng lượng phổ biến và vectơ trọng lượng mới là nhỏ, vectơ lỗi có thể được mở rộng theo thứ tự đầu tiên bằng một chuỗi Taylor.

$$
e_{(j+i)}=\frac{e_{(j)}+\partial e_{k}}{\partial \mathrm{w}_{i}}\left(\mathrm{w}_{(j+1)}-\mathrm{w}_{(j)}\right)
$$

Do đó, hàm lỗi có thể được biểu thị bằng:

$$
E=\frac{1}{2}\left\|\frac{e_{(j)}+\partial e_{k}}{\partial \mathrm{w}_{i}}\left(\mathrm{w}_{(j+1)}-\mathrm{w}_{(j)}\right)\right\|^{2}
$$

Tối thiểu hóa hàm lỗi đối với vectơ trọng lượng mới ta có:

$$
\mathrm{w}_{(j+1)}=\mathrm{w}_{(j)}-\left(Z^{T} Z\right)^{-1} Z^{T} e_{(j)}
$$

trong đó $(Z)_{k i} \equiv \frac{\partial e_{k}}{\partial \mathrm{w}_{i}}$

Hàm Hessian cho hàm lỗi tổng bình phương là:

$$
(H)_{\mathrm{ij}}=\frac{\partial^{2} E}{\partial \mathrm{w}_{i} \partial \mathrm{w}_{j}}=\sum\left\{\left(\frac{\partial e_{k}}{\partial \mathrm{w}_{i}}\right)\left(\frac{\partial e_{k}}{\partial \mathrm{w}_{i}}\right)+\frac{e_{k} \partial^{2} e_{k}}{\partial \mathrm{w}_{i} \partial \mathrm{w}_{j}}\right\}
$$


Bỏ qua số hạng thứ hai, hàm Hessian có thể được viết là:

$$
H=Z^{T} Z
$$

Do đó, cần cập nhật các trọng số liên quan đến hàm Hessian nghịch đảo hoặc xấp xỉ chúng cho các mạng phi tuyến. Hàm Hessian tương đối dễ tính toán, vì nó dựa trên các đạo hàm bậc nhất đối với các trọng số mạng có thể dễ dàng điều chỉnh bằng cách truyền ngược. Mặc dù công thức cập nhật có thể được áp dụng lặp đi lặp lại để giảm thiểu hàm lỗi, nhưng điều này có thể dẫn đến kích thước bước lớn, vì vậy sẽ làm mất hiệu lực xấp xỉ tuyến tính công thức trên. Trong thuật toán Levenberg - Marquest, hàm lỗi được giảm thiểu, trong khi kích thước bước được giữ ở mức nhỏ để đảm bảo tính hợp lệ của phép tính gần đúng tuyến tính. Điều này được thực hiện bằng cách sử dụng hàm lỗi đã sửa đổi của biểu mẫu.

$$
E=\frac{1}{2}\left\|\frac{e_{(j)}+\partial e_{k}}{\partial \mathrm{w}_{i}}\left(\mathrm{w}_{(j+1)}-\mathrm{w}_{j}\right)\right\|^{2}+\lambda\left\|\mathrm{w}_{(j+1)}-\mathrm{w}_{(j)}\right\|^{2}
$$

Trong đó $\lambda$ là tham số chi phối kích thước bước. Giảm thiểu lỗi đã sửa đổi liên quan đến $\mathrm{w}_{(\mathrm{j}+1)}$ cho ta

$$
\mathrm{w}_{(j+1)}=\mathrm{w}_{(j)}-\left(Z^{T} Z+\lambda I\right)^{-1} Z^{T} e(j)
$$

giá trị rất lớn của $\lambda$ tiến đến độ dốc tiêu chuẩn, trong khi giá trị rất nhỏ sát với phương pháp Newton.

\subsection{Thí nghiệm và dữ liệu sử dụng cho bài toán}

Nghiên cứu này sử dụng các cột ống thép nhồi bê tông tiết diện chữ nhật. Thí nghiệm xác định sức chịu tải tới hạn được minh họa trong Hình 2. Thí nghiệm nén ở trong bài toán được coi là nén dọc trục đúng tâm. Cần lưu ý rằng các khiếm khuyết ban đầu về hình học và các ứng suất dư trong quá trình chế tạo cấu kiện đã được bỏ qua. Một số công bố quốc tế cũng chỉ ra rằng các yếu tố này có rất ít ảnh hưởng tới sức chịu tải tới hạn của cấu kiện cột ống thép nhồi bê tông. Số liệu bao gồm 99 kết quả thử nghiệm trong nghiên cứu đã được công bố tại các tạp chí quốc tế uy tín của các tác giả Bridge [20], Du và cộng sự [21], Du và cộng sự [22], Ghannam và cộng sự [23], Han [24], Han \& Yang [25], Han \& Yao [26], Lin [27], Schneider [28], Shakir-Khalil \& Mouli [29] và Shakir-Khalil \& Zeghiche [30]. Bài toán mô phỏng sử dụng tổng cộng 6 tham số đầu vào cho mô hình $\mathrm{ANN}$ bao gồm: chiều cao mặt cắt cột $(\mathrm{H})$, chiều rộng của mặt cắt cột $(\mathrm{W})$, chiều dày của lớp thép bọc $(\mathrm{t})$, chiều dài của cột $(\mathrm{L})$, cường độ chịu nén của bê tông sử dụng $\left(\mathrm{f}^{\prime} \mathrm{c}\right)$ và cường độ của thép $\left(\mathrm{f}_{\mathrm{y}}\right)$. Tham số đầu ra được xét tới là sức chịu tải (sức kháng cột) tới hạn của cột $(\mathrm{Pu})$. Số dữ liệu này được chia thành 2 phần: Phần thứ nhất (70\% số liệu) dùng để huấn luyện mạng $\mathrm{ANN}$, được gọi là phần huấn luyện. Phần thứ hai ( $30 \%$ số liệu) dùng để kiểm chứng mô hình, sẽ được gọi tắt là phần kiểm chứng. Với cách chia trên, bộ dữ liệu gồm 99 số liệu sẽ có 69 số liệu dùng để huấn luyện và 30 số liệu dùng để đánh giá khả năng dự báo của mạng ANN. Tất cả các giá trị của bài toán, bao gồm đầu vào và hàm mục tiêu, được chuẩn hóa về khoảng giá trị từ 0 đến 1 để giảm thiểu sai số do mô phỏng tạo ra. Đây là một kỹ thuật được dùng rất nhiều trong các bài toán sử dụng trí thông minh nhân tạo để mô phỏng. 
(a)

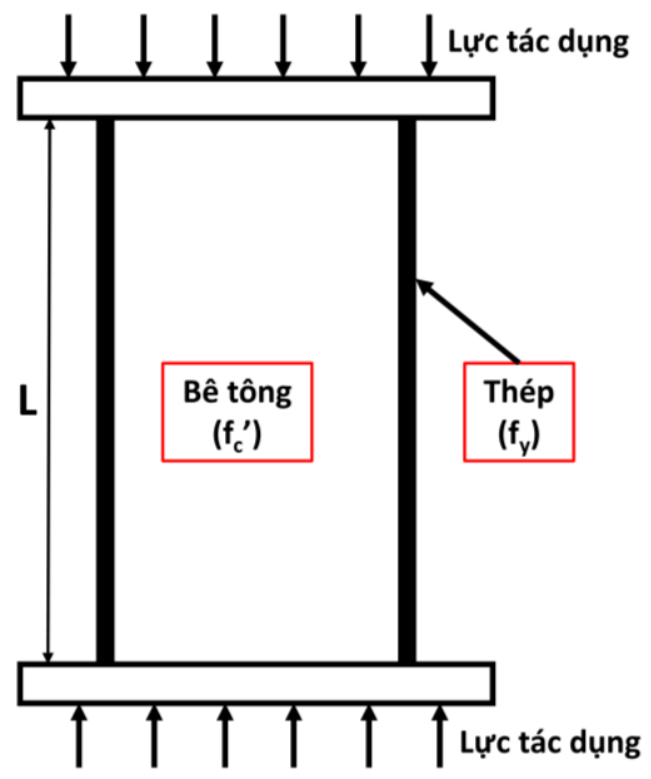

(b)

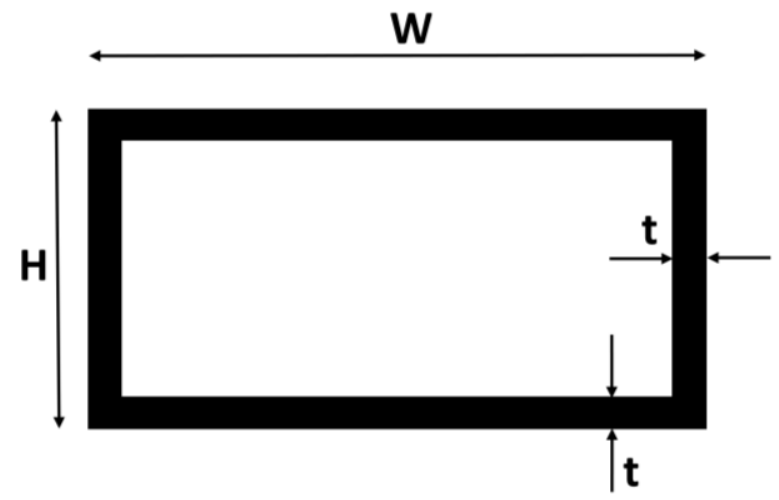

Hình 2. (a) Sơ đồ thí nghiệm xác định sức chịu tải tới hạn của cấu kiện cột ống thép nhồi bê tông tiết diện chữ nhật; (b) Sơ đồ mặt cắt ngang của cấu kiện.

\subsection{Các chỉ tiêu đánh giá năng lực dự báo}

Trong nghiên cứu này, các chỉ tiêu đánh giá phổ biến như hệ số tương quan Pearson $(\mathrm{R})$, sai số toàn phương trung bình (root mean square error - RMSE) và sai số tuyệt đối trung bình (mean absolute error - MAE) được sử dụng để đánh giá tương quan giữa các giá trị dự báo bằng mô hình $\mathrm{ANN}$ và giá trị thí nghiệm thực tế. Về cơ bản, MAE và RMSE càng tiến tới 0 thì mô hình có năng lực càng cao. Giá trị của $R$ nằm trong khoảng -1 đến 1 , và giá trị tuyệt đối của $R$ càng tiến tới 1 thì mô hình có độ chính xác càng cao. Công thức tính $R, R M S E$ và MAE có thể được tham khảo trong các tài liệu trích dẫn [31,32].

\section{KẾT QUẢ VÀ THẢO LUẬN}

Như đã trình bày ở phần tổng quan của vấn đề, mô hình dự báo bằng mạng Nơ-Ron nhân tạo có nhiều ưu điểm so với các phương pháp truyền thống như thí nghiệm hay mô phỏng bằng phần tử hữu hạn. Bản chất của việc tách $70 \%$ dữ liệu cho việc huấn luyện mạng $\mathrm{ANN}$ và để tách biệt phần kiểm chứng và huấn luyện. Nghĩa là dữ liệu của phần kiểm chứng $(30 \%)$ hoàn toàn không được mô hình ANN biết đến trước đó. Chính vì vậy, năng lực dự báo của mô hình $\mathrm{ANN}$ có thể được đánh giá một cách khách quan và chính xác nhất. Có thể so sánh điều này với việc dự báo hàm mục tiêu của các thí nghiệm tiếp theo (30\% dữ liệu chưa được mô hình học) dựa trên các kết quả thí nghiệm đã có sã̃n trước đó (70\% dự liệu đã được mồ hình học). Đối với các bài toán dự báo nói chung, năng lực dự báo của mô hình là quan trọng nhất. Nó được thể hiện thông qua các chỉ tiêu đánh giá sai số, như đã trình bày ở phần trước. Chính vì vậy, các kết quả ở những phần tiếp theo chỉ tập trung vào những chỉ tiêu đánh giá cho phần kiểm chứng mô hình.

\subsection{Nghiên cứu xác định số No-Ron tối ưu cho ANN}

Trong phần này, tác giả sẽ trình bày kết quả của việc sử dụng mô hình ANN trong việc dự báo khả năng chịu tải tới hạn của cấu kiện cột ống thép nhồi bê tông có tiết diện hình chữ 
nhật. Cấu trúc của mô hình ANN trong dự báo là một nhân tố quan trọng, ảnh hưởng lớn đến khả năng dự báo của mô hình. Chính vì vậy, tổng cộng 6000 mô phỏng đã được thực hiện ở phần này, bao gồm việc cho biến thiên số Nơ-Ron từ 1 đến 20 và chạy 300 mô phỏng cho mỗi trường hợp như vậy. Lý do của việc chạy 300 lần là để chắc chắn việc hội tụ của kết quả và kiểm tra kỹ năng lực dự báo của mô hình. Hình 3 biểu thị kết quả của mô phỏng xem xét ảnh hưởng của số Nơ-Ron tới kết quả dự báo, với "median" được định nghĩa là số trung vị, 25\%75\% được hiểu là giá trị trong khoảng tứ phân vị thứ nhất và tứ phân vị thứ ba, $\mu$ là giá trị trung bình và $\sigma$ là độ lệch chuẩn. Kết quả cho thấy, xét theo giá trị của RMSE thì số Nơ-Ron lấy theo giá trị 4 sẽ có sai số ít nhất, và tương tự giá trị của $\mathrm{R}$ cao nhất. Ngoài ra, nếu lấy theo tiêu chí MAE thì số Nơ-Ron là 7 sẽ cho sai số thấp nhất. Tuy nhiên, số Nơ-Ron từ 4 đến 7 thì độ lệch của kết quả trung bình của MAE không chênh lệch quá lớn. Khi giá trị của số Nơ-Ron nằm ngoài khoảng 4-7 thì có thể thấy sai số của bài toán lớn dần lên, nghĩa là RMSE, MAE tăng và $\mathrm{R}$ giảm. Hơn nữa, có thể thấy rằng giá trị trung bình và độ lệch chuẩn của trường hợp 4 Nơ-Ron là dao động bé nhất. Chính vì vậy, nhóm tác giả chọn số Nơ-Ron là 4 cho lớp ẩn ở giữa để tiến hành các nghiên cứu tiếp theo đối với bài toán dự báo khả năng chịu lực dọc trục tới hạn của cột ống thép nhồi bê tông có tiết diện hình chữ nhật.

(a)

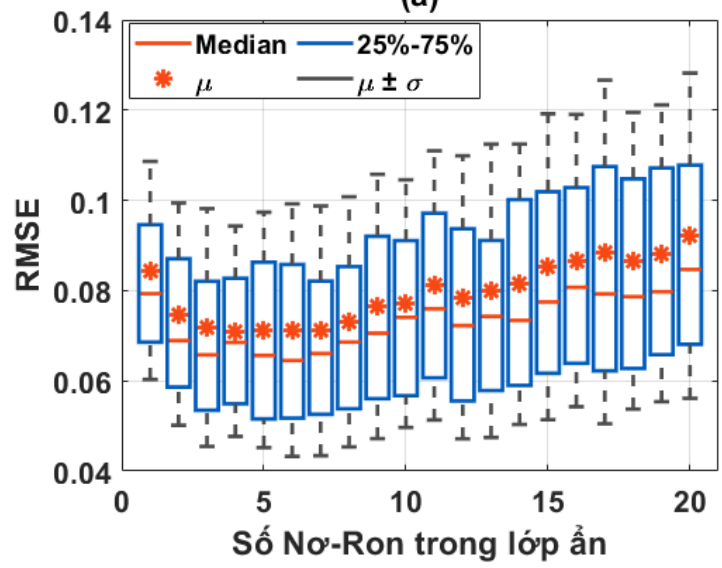

(b)

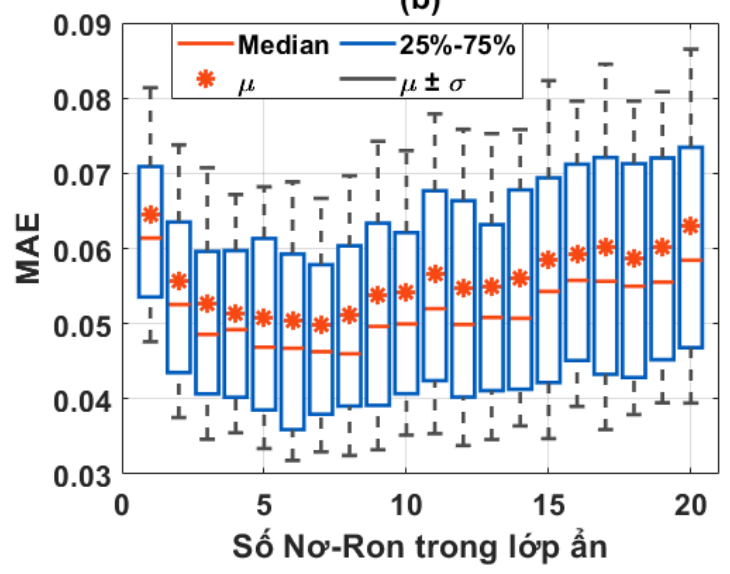

(c)

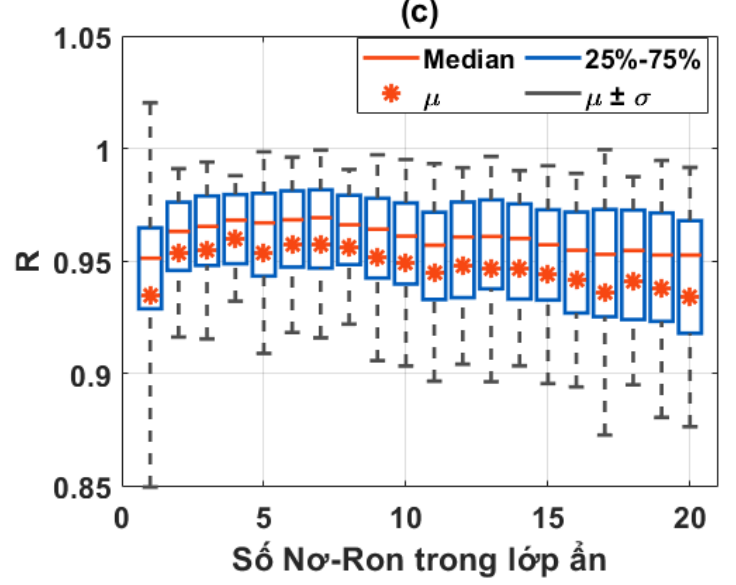

Hình 3. Ảnh hưởng của số Nơ-Ron tới kết quả dự báo của ANN tính cho 300 lần mô phỏng trong mỗi trường hợp, đánh giá bằng các chỉ tiêu: (a) RMSE; (b) MAE; (c) R.

\subsection{Kết quả điển hình về dụ báo khả năng chịu tải tới hạn của cột ống thép nhồi bê tông}

Sau khi đã chọn được số Nơ-Ron thích hợp cho bài toán dự báo, nhóm tác giả tiến hành phân tích kết quả thu được đối với trường hợp sử dụng 4 Nơ-Ron cho lớp ẩn ở giữa. Cấu trúc 
ANN của bài toán này có thể được viết dưới dạng ANN-[6-4-1], nghĩa là cấu trúc ANN có 6 biến đầu vào, 4 Nơ-Ron ở lớp ẩn, và 1 biến đầu ra. Hình 4 biểu thị biểu đồ tần suất và hàm mật độ xác suất cho 300 lần chạy mô phỏng dự báo khả năng chịu lực dọc trục tới hạn. Đối với chỉ tiêu RMSE, sai số tập trung lớn nhất ở khoảng 0.05-0.08 với hàm mật độ xác suất rất tập trung cao ở khoảng này. Sai số bé nhất trong 300 lần chạy mô phỏng có thể đạt tới 0.02 , trong khi cũng có một vài trường hợp cho sai số cao, khoảng 0.2. Những nhận định tương tự cho MAE có thể được quan sát trên biểu đồ, với khoảng sai số tập trung ở 0.03 đến 0.06 . Ngoài ra, với hệ số tương quan $\mathrm{R}$, mô hình $\mathrm{ANN}-[6-4-1]$ cho tương quan rất tốt giữa kết quả thí nghiệm và kết quả mô phỏng với $\mathrm{R}$ tập trung ở khoảng giá trị $\mathrm{R}=0.96$ đến 0.99 , nghĩa là khả năng dự báo gần như là chính xác tuyệt đối.

Nhóm tác giả trình bày trong Hình 5 một kết quả điển hình trong dự báo sức chịu tải tới hạn của cấu kiện cột ống thép nhồi bê tông, đây là kết quả dự báo tốt nhất trong 300 lần chạy mô phỏng. Đối với bộ dữ liệu huyến luyện, tương quan giữa kết quả mô phỏng và thí nghiệm đạt đến $\mathrm{R}=0.99284$, cho thấy năng lực dự báo của mô hình là rất tốt. Có thể nhận thấy rằng kết quả sai số chủ yếu nằm ở những giá trị chịu tải tới hạn lớn. Đối với bộ dữ liệu kiểm chứng mô hình, kết quả tương quan $\mathrm{R}$ đạt được là $\mathrm{R}=0.99727$ và sai số tập trung chủ yếu ở phần tư vị thứ nhất. Đối với bộ dữ liệu huyến luyện, hàm số " $\mathrm{y}=0.99 \mathrm{x}+0.0036$ " được thiết lập để thể hiện tương quan giữa dữ liệu thực nghiệm và dữ liệu mô phỏng. Tương tự, hàm số " $\mathrm{y}=0.99 \mathrm{x}+$ 0.0012 " được thiết lập cho tương quan ở bộ dữ liệu kiểm chứng.

(a)

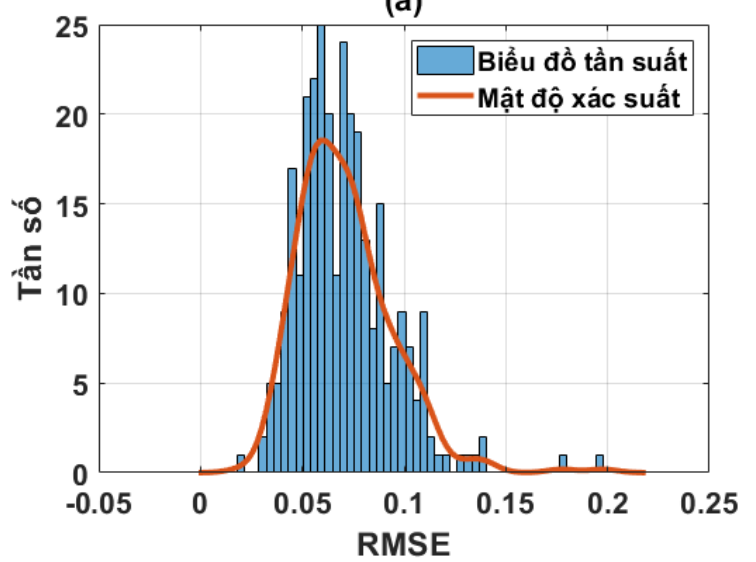

(b)

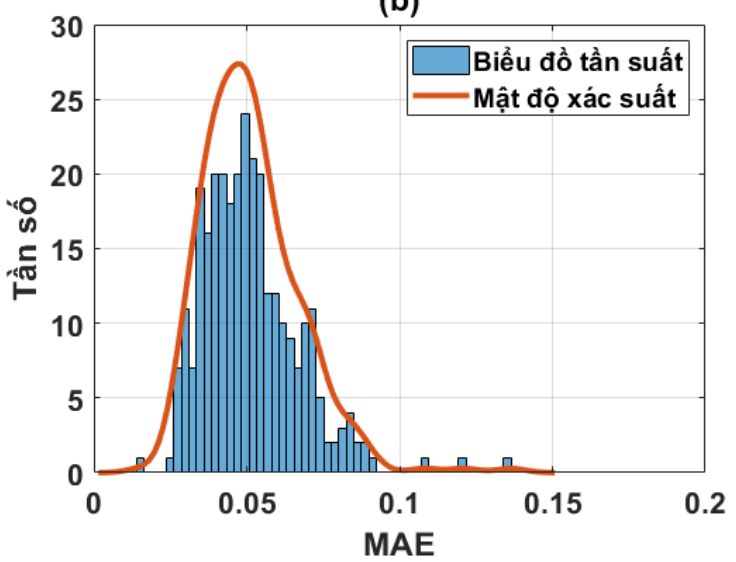

(c)

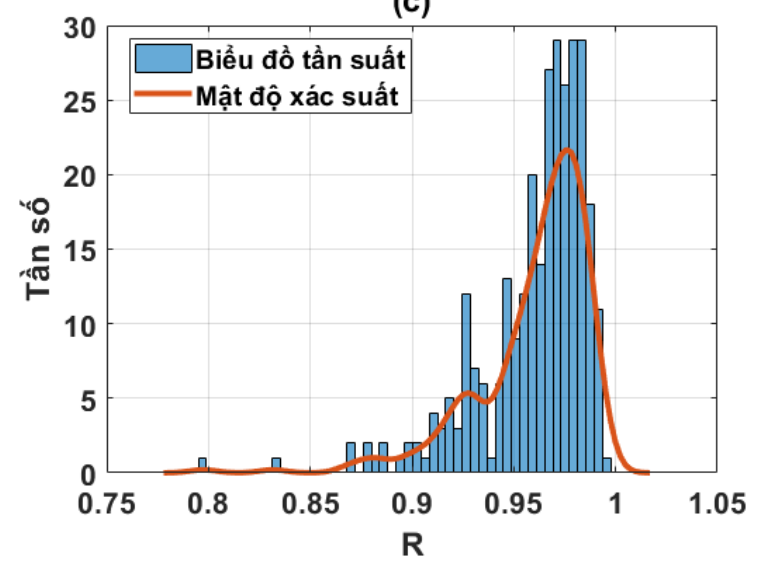

Hình 4. Biểu đồ tần suất và hàm mật độ xác suất cho trường hợp mô phỏng bằng 4 Nơ-Ron cho 300 lần chạy mô phỏng dự báo sức chịu tải tới hạn của cấu kiện cột ống thép nhồi bê tông. 
(a)

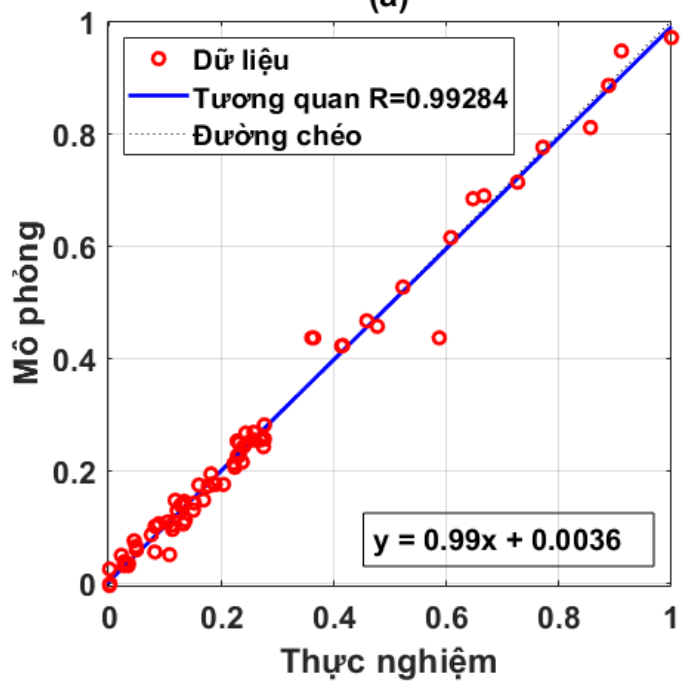

(b)

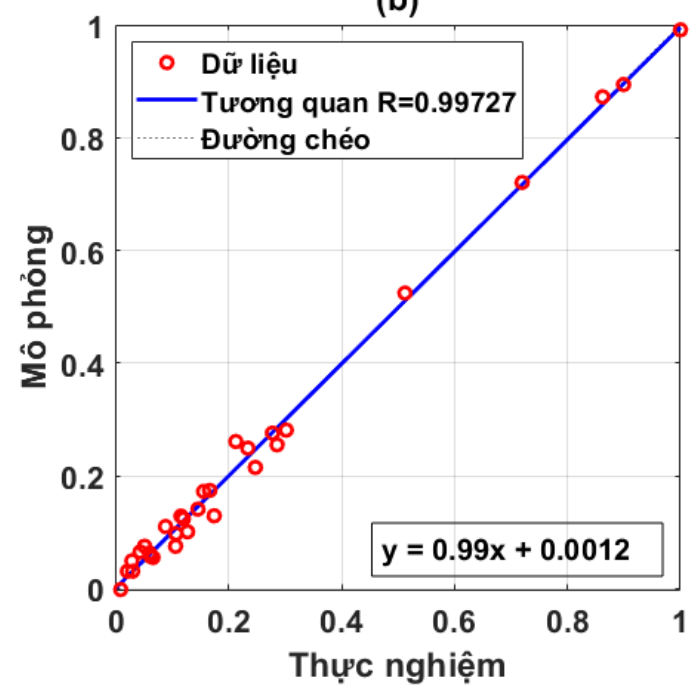

Hình 5. So sánh tương quan giữa bộ dữ liệu huấn luyện và kiểm chứng.

Để minh chứng cho tính chính xác của mô hình $\mathrm{ANN}$, nhóm tác giả trình bày trong Bảng 1 bộ số liệu 30 mẫu thí nghiệm và so sánh với tính toán của hai tiêu chuẩn EC4 (2004) và ACI (2005). Cần lưu ý rằng, số liệu này sau khi đã mô phỏng bằng $\mathrm{ANN}$ đã được quy lại về giá trị thực. Các công thức của hai tiêu chuẩn EC4 (2004) và ACI (2005) có thể được tham khảo tại tài liệu [33]. Có thể thấy rằng kết quả mô phỏng bằng $\mathrm{ANN}$ cho sai số rất thấp ở trong hầu hết các trường hợp. Có 2 trường hợp $\mathrm{ANN}$ cho kết quả có sai số nhiều hơn so với hai tiêu chuẩn EC4 và ACI. Ngoài ra, có 1 mẫu thí nghiệm ANN mô phỏng cho sai số lớn hơn ACI nhưng tốt hơn $\mathrm{EC} 4$, và 1 mẫu cho sai số lớn hơn EC4 nhưng tốt hơn $\mathrm{ACI}$.

\section{KẾT LUẬN}

Trong bài báo này, sử dụng tập hợp các kết quả thí nghiệm được thực hiện trên các cột CFST hình chữ nhật chịu tải trọng dọc trục, một mạng lưới thần kinh nhân tạo đã được đề xuất là mạng nơ ron lan truyền ngược dung thuật toán Levenberg - Marquart. Dữ liệu đầu vào cho đào tạo mạng là: chiều cao mặt cắt cột $(\mathrm{H})$, chiều rộng của mặt cắt cột $(\mathrm{W})$, chiều dày của lớp thép bọc $(\mathrm{t})$, chiều dài của cột $(\mathrm{L})$, cường độ chịu nén của bê tông sử dụng $\left(\mathrm{f}^{\prime} \mathrm{c}\right)$ và cường độ của thép $\left(\mathrm{f}_{\mathrm{y}}\right)$. Ba chỉ tiêu hệ số tương quan $(\mathrm{R})$, sai số toàn phương trung bình (RMSE) và sai số tuyệt đối trung bình (MAE) được sử dụng để đánh giá tương quan giữa các giá trị dự báo bằng mô hình $\mathrm{ANN}$ và giá trị thí nghiệm thực tế. Sau khi đào tạo các mạng với số lượng tế bào thần kinh ẩn khác nhau, số lượng các tế bào thần kinh ở lớp ẩn là bốn đã được chọn. Sau đó, cấu trúc mạng [6-4-1] để xác định cường độ nén của bê tông trong các cột CFST đã được trình bày. Kết quả của nghiên cứu chỉ ra rằng mô hình đề xuất có khả năng dự đoán sức chịu tải tới hạn cho cột CFST hình chữ nhật với độ chính xác tốt, tương quan $\mathrm{R}$ có thể lên tới $\mathrm{R}=0.99727$.

Do sử dụng 99 kết quả thử nghiệm nên kết quả của bài báo này chỉ có độ tin cậy cao trong phạm vi các số liệu được thí nghiệm. Chính vì vậy, hướng nghiên cứu tiếp theo của nhóm tác giả sẽ bao gồm: (i) tiến hành thí nghiệm thêm các trường hợp khác để mở rộng phạm vi của những yếu tố đầu vào và (ii) tiến hành thu thập thêm những số liệu về cấu kiện có mặt cắt hình chữ nhật để kiểm chứng năng lực dự báo của mô hình. 
Transport and Communications Science Journal, Vol 71, Issue 2 (02/2020), 154-166

Bảng 1. Kết quả so sánh giữa mô phỏng ANN và các tiêu chuẩn EC4 (2004) và ACI (2005).

\begin{tabular}{|c|c|c|c|c|c|c|c|c|c|c|c|c|}
\hline $\mathbf{H}$ & $\mathbf{W}$ & $\mathbf{t}$ & $\mathbf{L}$ & $\mathbf{f}_{\mathbf{y}}$ & $\mathbf{f}_{\mathbf{c}}$ & $\mathbf{P u}$ & ANN & EC4 & ACI & $\begin{array}{c}\text { Err } \\
(\mathrm{ANN})\end{array}$ & $\begin{array}{c}\text { Err } \\
(\text { EC4) }\end{array}$ & $\begin{array}{c}\text { Err } \\
(\mathrm{ACI})\end{array}$ \\
\hline 121.2 & 70.9 & 3.4 & 401.2 & 231.0 & 46.9 & 815.0 & 720.6 & 591.9 & 538.7 & $11.6 \%$ & $27.4 \%$ & $33.9 \%$ \\
\hline 141.9 & 76.4 & 3.5 & 2333.3 & 331.2 & 26.4 & 552.0 & 584.0 & 665.6 & 627.7 & $-5.8 \%$ & $-20.6 \%$ & $-13.7 \%$ \\
\hline 90.0 & 60.0 & 3.4 & 307.7 & 231.0 & 46.9 & 1210.0 & 1258.0 & 397.1 & 364.7 & $-4.0 \%$ & $67.2 \%$ & $69.9 \%$ \\
\hline 235.4 & 109.1 & 3.1 & 744.0 & 353.1 & 17.4 & 576.0 & 640.7 & 1042.7 & 980.3 & $-11.2 \%$ & $-81.0 \%$ & $-70.2 \%$ \\
\hline 144.4 & 89.2 & 5.1 & 655.7 & 432.5 & 26.0 & 645.0 & 720.6 & 1105.3 & 1062.3 & $-11.7 \%$ & $-71.4 \%$ & $-64.7 \%$ \\
\hline 118.0 & 116.6 & 5.4 & 655.7 & 333.4 & 23.8 & 968.0 & 1021.0 & 902.5 & 860.0 & $-5.5 \%$ & $6.8 \%$ & $11.2 \%$ \\
\hline 188.7 & 120.0 & 3.1 & 2426.8 & 353.1 & 17.4 & 1370.0 & 1276.1 & 904.0 & 848.9 & $6.9 \%$ & $34.0 \%$ & $38.0 \%$ \\
\hline 193.8 & 87.3 & 6.0 & 2073.6 & 374.7 & 26.4 & 844.0 & 885.9 & 1411.7 & 1355.7 & $-5.0 \%$ & $-67.3 \%$ & $-60.6 \%$ \\
\hline 141.9 & 87.3 & 6.0 & 3050.0 & 360.3 & 38.5 & 3150.0 & 3180.9 & 1168.5 & 1109.4 & $-1.0 \%$ & $62.9 \%$ & $64.8 \%$ \\
\hline 193.8 & 87.3 & 6.0 & 2073.6 & 374.7 & 7.9 & 580.0 & 584.0 & 1150.2 & 1133.4 & $-0.7 \%$ & $-98.3 \%$ & $-95.4 \%$ \\
\hline 193.8 & 141.8 & 0.7 & 827.1 & 249.7 & 22.6 & 1251.0 & 1152.5 & 704.1 & 612.3 & $7.9 \%$ & $43.7 \%$ & $51.1 \%$ \\
\hline 90.0 & 65.5 & 3.4 & 619.4 & 302.3 & 27.1 & 691.0 & 658.9 & 385.6 & 365.0 & $4.6 \%$ & $44.2 \%$ & $47.2 \%$ \\
\hline 360.0 & 240.0 & 3.1 & 1491.9 & 353.1 & 17.4 & 880.0 & 799.9 & 2493.2 & 2275.7 & $9.1 \%$ & $-183.3 \%$ & $-158.6 \%$ \\
\hline 144.4 & 89.6 & 8.9 & 655.7 & 372.6 & 23.8 & 1420.0 & 1356.7 & 1422.5 & 1387.7 & $4.5 \%$ & $-0.2 \%$ & $2.3 \%$ \\
\hline 141.9 & 76.4 & 3.5 & 2333.3 & 331.2 & 7.9 & 515.0 & 484.0 & 488.3 & 476.9 & $6.0 \%$ & $5.2 \%$ & $7.4 \%$ \\
\hline 193.8 & 141.8 & 0.7 & 494.7 & 249.7 & 22.6 & 2710.0 & 2712.4 & 704.1 & 612.3 & $-0.1 \%$ & $74.0 \%$ & $77.4 \%$ \\
\hline 188.7 & 120.0 & 3.1 & 806.3 & 353.1 & 17.4 & 2069.0 & 2106.9 & 904.0 & 848.9 & $-1.8 \%$ & $56.3 \%$ & $59.0 \%$ \\
\hline 141.9 & 76.4 & 3.5 & 2333.3 & 331.2 & 26.4 & 3575.0 & 3547.9 & 665.6 & 627.7 & $0.8 \%$ & $81.4 \%$ & $82.4 \%$ \\
\hline 110.8 & 65.5 & 6.0 & 203.9 & 354.1 & 40.5 & 760.0 & 828.7 & 809.8 & 775.7 & $-9.0 \%$ & $-6.5 \%$ & $-2.1 \%$ \\
\hline 131.5 & 92.7 & 3.4 & 432.4 & 231.0 & 46.9 & 672.0 & 683.3 & 787.1 & 709.6 & $-1.7 \%$ & $-17.1 \%$ & $-5.6 \%$ \\
\hline 175.2 & 111.3 & 10.0 & 558.1 & 514.5 & 43.7 & 857.9 & 871.3 & 2932.6 & 2833.9 & $-1.6 \%$ & $-241.8 \%$ & $-230.3 \%$ \\
\hline 188.7 & 120.0 & 3.1 & 806.3 & 353.1 & 17.4 & 620.8 & 690.1 & 904.0 & 848.9 & $-11.2 \%$ & $-45.6 \%$ & $-36.7 \%$ \\
\hline 152.3 & 109.1 & 9.2 & 494.7 & 194.0 & 46.9 & 1144.1 & 1294.3 & 1312.5 & 1221.2 & $-13.1 \%$ & $-14.7 \%$ & $-6.7 \%$ \\
\hline 110.8 & 76.4 & 3.4 & 619.4 & 302.3 & 27.1 & 1026.1 & 887.7 & 504.8 & 474.5 & $13.5 \%$ & $50.8 \%$ & $53.8 \%$ \\
\hline 110.8 & 65.5 & 5.4 & 3050.0 & 403.4 & 34.8 & 679.8 & 664.5 & 798.9 & 768.8 & $2.3 \%$ & $-17.5 \%$ & $-13.1 \%$ \\
\hline 197.0 & 88.4 & 10.0 & 626.6 & 514.5 & 34.1 & 817.1 & 789.2 & 2817.0 & 2751.5 & $3.4 \%$ & $-244.7 \%$ & $-236.7 \%$ \\
\hline 141.9 & 87.3 & 6.0 & 203.9 & 360.3 & 38.3 & 937.1 & 923.9 & 1166.5 & 1107.6 & $1.4 \%$ & $-24.5 \%$ & $-18.2 \%$ \\
\hline 131.5 & 92.7 & 3.4 & 432.4 & 231.0 & 46.9 & 1001.5 & 1026.4 & 787.1 & 709.6 & $-2.5 \%$ & $21.4 \%$ & $29.1 \%$ \\
\hline 141.9 & 87.3 & 6.0 & 100.0 & 353.0 & 38.7 & 3264.0 & 3248.5 & 1154.9 & 1095.4 & $0.5 \%$ & $64.6 \%$ & $66.4 \%$ \\
\hline 110.8 & 76.4 & 3.4 & 370.1 & 231.0 & 46.9 & 1344.6 & 1340.2 & 576.2 & 523.6 & $0.3 \%$ & $57.1 \%$ & $61.1 \%$ \\
\hline
\end{tabular}

\section{TÀI LIỆU THAM KHẢO}

[1] M. Javed, N.H. Ramli Sulong, N. Khan, S. Kashif, Finite element analysis of the flexural behavior of square CFST beams at ambient and elevated temperature, in: Proceedings of the 12th International Conference on Advances in Steel-Concrete Composite Structures. ASCCS 2018, Editorial Universitat Politècnica de València, 2018, pp. 843-850.

[2] Z. Tian, Y. Liu, L. Jiang, W. Zhu, Y. Ma, A review on application of composite truss bridges composed of hollow structural section members, Journal of Traffic and Transportation Engineering (English Edition), 6 (2019) 94-108. https://doi.org/10.1016/j.jtte.2018.12.001

[3] D.M. Lue, J.-L. Liu, T. Yen, Experimental study on rectangular CFT columns with high-strength 
Tạp chí Khoa học Giao thông vận tải, Tập 71, Số 2 (02/2020), 154-166

concrete, Journal of Constructional Steel Research, 63 (2007) 37-44. https://doi.org/10.1016/j.jcsr.2006.03.007

[4] L.-H. Han, Tests on stub columns of concrete-filled RHS sections, Journal of Constructional Steel Research, 58 (2002) 353-372. https://doi.org/10.1016/S0143-974X(01)00059-1

[5] J. Zeghiche, K. Chaoui, An experimental behaviour of concrete-filled steel tubular columns, Journal of Constructional Steel Research, 61 (2005) 53-66. https://doi.org/10.1016/j.jcsr.2004.06.006

[6] L.-H. Han, Y.-F. Yang, Influence of concrete compaction on the behavior of concrete filled steel tubes with rectangular sections, Advances in Structural Engineering, 4 (2001) 93-100. https://doi.org/10.1260/1369433011502381

[7] X. Wang, Y. Qi, Y. Sun, Z. Xie, W. Liu, Compressive behavior of composite concrete columns with encased FRP confined concrete cores, Sensors, $19 \quad$ (2019) 1792. https://doi.org/10.3390/s19081792

[8] Z. Tao, Z.-B. Wang, Q. Yu, Finite element modelling of concrete-filled steel stub columns under axial compression, Journal of Constructional Steel Research, 89 (2013) 121-131. https://doi.org/10.1016/j.jcsr.2013.07.001

[9] D. Liu, W.-M. Gho, Axial load behaviour of high-strength rectangular concrete-filled steel tubular stub columns, Thin-Walled Structures, 43 (2005) 1131-1142. https://doi.org/10.1016/j.tws.2005.03.007

[10] D. Liu, Tests on high-strength rectangular concrete-filled steel hollow section stub columns, Journal of Constructional Steel Research, $61 \quad$ (2005) 902-911. https://doi.org/10.1016/j.jcsr.2005.01.001

[11] D. Liu, W.-M. Gho, J. Yuan, Ultimate capacity of high-strength rectangular concrete-filled steel hollow section stub columns, Journal of Constructional Steel Research, 59 (2003) 1499-1515. https://doi.org/10.1016/S0143-974X(03)00106-8

[12] Eurocode 4, Design of composite steel and concrete structures. Part 1.1, General rules and rules for buildings, European Committee for Standardization, British Standards Institution, London, UK, 2004.

[13] AISC, Load and resistance factor design (LRFD) specification for structural steel buildings, American Institute of Steel Construction, Chicago, USA, 2005.

[14] A.C.I. Committee, Building code requirements for structural concrete (ACI 318-05) and commentary (ACI 318R-05), in: American Concrete Institute, 2005.

[15] A.C.I. Committee, Building code requirements for structural concrete (ACI 318-08) and commentary, in: American Concrete Institute, 2008.

[16] X.B. Ma, S.M. Zhang, Comparison of design methods of load-carrying capacity for circular concrete-filled steel tube beam columns in typical codes worldwide, Journal of Harbin Institute of Technology, 39 (2007) 536-541.

[17] H.-B. Ly, L.M. Le, H.T. Duong, T.C. Nguyen, T.A. Pham, T.-T. Le, V.M. Le, L. Nguyen-Ngoc, B.T. Pham, Hybrid Artificial Intelligence Approaches for Predicting Critical Buckling Load of Structural Members under Compression Considering the Influence of Initial Geometric Imperfections, Applied Sciences. 9 (2019) 2258. https://www.mdpi.com/2076-3417/9/11/2258

[18] H.-B. Ly, B.T. Pham, D.V. Dao, V.M. Le, L.M. Le, T.-T. Le, Improvement of ANFIS Model for Prediction of Compressive Strength of Manufactured Sand Concrete, Applied Sciences, 9 (2019) 3841. https://www.mdpi.com/2076-3417/9/18/3841

[19] D.V. Dao, H. Adeli, H.-B. Ly, L.M. Le, V.M. Le, T.-T. Le, B.T. Pham, A Sensitivity and Robustness Analysis of GPR and ANN for High-Performance Concrete Compressive Strength Prediction Using a Monte Carlo Simulation, Sustainability, 12 (2020) 830. https://doi.org/10.3390/su12030830

[20] R.Q. Bridge, Concrete filled steel tubular columns / by R.Q. Bridge., School of Civil Engineering, University of Sydney, Sydney, Australia, 1976. https://trove.nla.gov.au/version/44129332

[21] Y. Du, Z. Chen, M.-X. Xiong, Experimental behavior and design method of rectangular concretefilled tubular columns using Q460 high-strength steel, Construction and Building Materials, 125 (2016) 856-872. https://doi.org/10.1016/j.conbuildmat.2016.08.057 
Transport and Communications Science Journal, Vol 71, Issue 2 (02/2020), 154-166

[22] Y. Du, Z. Chen, Y. Yu, Behavior of rectangular concrete-filled high-strength steel tubular columns with different aspect ratio, Thin-Walled Structures, 109 (2016) 304-318. https://doi.org/10.1016/j.tws.2016.10.005

[23] S. Ghannam, Y.A. Jawad, Y. Hunaiti, Failure of lightweight aggregate concrete-filled steel tubular columns, Steel and Composite Structures, 4 (2004) 1-8. https://doi.org/10.12989/scs.2004.4.1.001

[24] L.-H. Han, Tests on stub columns of concrete-filled RHS sections, Journal of Constructional Steel Research, 58 (2002) 353-372. https://doi.org/10.1016/S0143-974X(01)00059-1.

[25] L.-H. Han, Y.-F. Yang, Analysis of thin-walled steel RHS columns filled with concrete under long-term sustained loads, Thin-Walled Structures. 41 (2003) 849-870. https://doi.org/10.1016/S0143974X(01)00059-1

[26] L.-H. Han, G.-H. Yao, Influence of concrete compaction on the strength of concrete-filled steel RHS columns, Journal of Constructional Steel Research, 59 (2003) 751-767. https://doi.org/10.1016/S0143-974X(02)00076-7

[27] C.Y. Lin, Axial Capacity of Concrete Infilled Cold-formed Steel Columns, in: Ninth International Specialty Conference on Cold-Formed Steel Structures, St. Louis, Missouri, U.S.A, 1988: pp. 443457.

[28] Schneider Stephen P., Axially Loaded Concrete-Filled Steel Tubes, Journal of Structural Engineering, 124 (1998) 1125-1138. https://doi.org/10.1061/(ASCE)0733-9445(1998)124:10(1125)

[29] H. Shakir-Khalil, M. Mouli, Further Tests on Concrete-Filled Rectangular Hollow-Section Columns, The Structural Engineer, 68 (1990) 405-413.

[30] H. Shakir-Khalil, J. Zeghiche, Experimental Behaviour of Concrete-Filled Rolled Rectangular Hollow-Section Columns, The Structural Engineer, 67 (1989) 346-353.

[31] H.-B. Ly, E. Monteiro, T.-T. Le, V.M. Le, M. Dal, G. Regnier, B.T. Pham, Prediction and Sensitivity Analysis of Bubble Dissolution Time in 3D Selective Laser Sintering Using Ensemble Decision Trees, Materials, 12 (2019) 1544. https://doi.org/10.3390/ma12091544

[32] H.-B. Ly, C. Desceliers, L.M. Le, T.-T. Le, B.T. Pham, L. Nguyen-Ngoc, V.T. Doan, M. Le, Quantification of Uncertainties on the Critical Buckling Load of Columns under Axial Compression with Uncertain Random Materials, Materials, 12 (2019) 1828. https://doi.org/10.3390/ma12111828

[33] Y. Du, Z. Chen, C. Zhang, C. Xiaochun, Research on axial bearing capacity of rectangular concrete-filled steel tubular columns based on artificial neural networks, Frontiers of Computer Science, 11 (2017) 863-873. https://doi.org/10.1007/s11704-016-5113-6 